\title{
HIPERTENSÃO PORTOPULMONAR EM TRANSPLANTE HEPÁTICO: RELATO DE CASO
}

\author{
Portopulmonary hypertension in liver transplantation: case report.
}

José Carlos Rodrigues Nascimento, ,2, Aline Menezes Sampaio², Tayná de Lima Freire², Anderson Emanuel Caliope Castelo Branco Mourão², Antonio Tiago Mota Pinheiro², Felipe Barbosa Lima², David Silveira Marinho ${ }^{1,2}$, Anderson da Silva Costa².

\section{RESUMO}

Introdução: A existência de hipertensão pulmonar associada à hipertensão portal é condição relevante em pacientes cirróticos. Na forma grave, é uma contraindicação absoluta para o transplante hepático. Relatamos um caso de hipertensão portopulmonar em transplante hepático, com pico hipertensivo severo durante reperfusão do enxerto hepático. Relato de caso: Paciente, sexo feminino, 40 anos, capacidade funcional < 4 METs, ASA IV, portadora de hepatopatia alcoólica com hipertensão portopulmonar, insuficiência renal dialítica e cardíaca. Foi evidenciada, no ecodopplercardiograma, pressão sistólica da artéria pulmonar estimada em $30 \mathrm{mmHg}$, sendo indicado cateterismo cardíaco direito, que mostrou pressão média de $48 \mathrm{mmHg}$ na artéria pulmonar (PMAP). A paciente foi submetida a transplante hepático sob anestesia geral e monitorizada com cateter Swan-Ganz ${ }^{\circledR}$, que mostrou PMAP de $28 \mathrm{mmHg}$ e pressão venosa central de $24 \mathrm{mmHg}$. A gasometria na hepatectomia estava normal. A fase anepática cursou sem sangramento considerável e com discreta oscilação da PMAP. Na reperfusão hepática, foi registrada maior variação da PMAP (26-50 mmHg), perdurando por 40 minutos, até o retorno aos valores iniciais, com conduta expectante. Até esse momento, foram ministradas $900 \mathrm{ml}$ de solução plasma-lyte albuminado a $2 \%$. Gasometria arterial após reperfusão, evidenciou acidose metabólica e respiratória com $\mathrm{pH}$ de 7,28 . No final da cirurgia, a paciente estava hemodinamicamente estável com PMAP de $30 \mathrm{mmHg}$, tendo sido encaminhada entubada à UTI. O volume total infundido foi $1650 \mathrm{ml}$. A paciente foi submetida a transplante renal 24 horas após o transplante hepático e recebeu alta hospitalar sem complicações. Discussão: O manejo anestésico intraoperatório requer o conhecimento das variações da pressão da artéria pulmonar nas fases do transplante hepático. A adequada monitorização do débito cardíaco, da variação do volume sistólico e da pressão de artéria pulmonar permitiram a otimização do uso de drogas vasoativas e da reposição de fluidos. Apesar da restrição hídrica ser descrita como forma adequada de controlar a sobrecarga volêmica na fase de reperfusão hepática, que é fator de instabilidade cardiovascular nesses pacientes, nesse caso, essa medida de restrição volêmica adotada, sem o uso de droga vasodilatadora pulmonar, não foi capaz de prevenir o aumento súbito da PMAP na reperfusão do enxerto.

Descritores: Hipertensão Pulmonar; Hipertensão Portal; Transplante de Fígado.

Instituição:

1 Unidade de Transplante Hepático do Hospital Geral de Fortaleza HGF, Fortaleza/CE, Brasil.

2 Serviço de Anestesiologia do Hospital Geral de Fortaleza - HGF, Fortaleza/CE, Brasil.

Correspondência:

José Carlos Rodrigues Nascimento

Rua Antonele Bezerra, 280, Apto.202, CEP 60160-070, Fortaleza/CE.

Tel.: (85) 99668-8500.

E-mail: jcr.nascimento@hotmail.com

Recebido em: 06/01/2016

Aceito em: 02/02/2016

\section{INTRODUÇÃO}

A hipertensão portopulmonar (HPP) é definida como hipertensão arterial pulmonar na presença de hipertensão portal independente do grau de comprometimento da função hepática. ${ }^{1}$

A HPP é uma complicação relevante da cirrose. Seu diagnóstico é importante, uma vez que define uma condição grave, que pode determinar pior prognóstico para o transplante hepático, sendo uma contraindicação a esse procedimento nas formas moderadas ou graves da doença. Esse quadro pessimista modificou-se recentemente com a possibilidade de tratar os pacientes com HPP com vasodilatadores específicos e reavaliá-los 
hemodinamicamente antes da contraindicação definitiva ao transplante hepático. ${ }^{2}$

A associação de hipertensão pulmonar com cirrose e hipertensão portal foi primeiramente descrita como um subtipo de hipertensão pulmonar primária em 1981. ${ }^{3}$ Passou a ser reconhecida como secundária em 1993 e denominada, a partir de então, como HPP. ${ }^{4}$ Ela é responsável por 7 a $10 \%$ de todos os casos de hipertensão pulmonar. ${ }^{5}$ Seu conceito envolve a exclusão de outras causas de hipertensão pulmonar secundária e é definida como pressão média na arterial pulmonar (PMAP) $>25 \mathrm{mmHg}$, pressão capilar pulmonar $(\mathrm{PCP})<$ $15 \mathrm{mmHg}$ e resistência vascular pulmonar $(\mathrm{RVP})>250$ dinas $/ \mathrm{s} / \mathrm{cm}^{5}$. ${ }^{6}$

Há grande discrepância nas estimativas de sobrevida publicadas de pacientes com HPP. Em um estudo americano, pacientes com HPP têm mal prognóstico, com taxa de sobrevida em três anos de $40 \% .^{7}$ Já em um estudo francês, a sobrevida foi de $68 \%$ em três anos. ${ }^{8}$ Esses resultados discordantes são susceptíveis, explicados por diferenças importantes no que diz respeito à gravidade da doença hepática, tendo a pesquisa francesa mais pacientes com cirrose leve.

HPP em transplante de fígado na doença leve (PMAP $<35 \mathrm{mmHg}$ ) representa pouco risco perioperatório, ${ }^{9}$ mas a doença moderada (PMAP $35-45 \mathrm{mmHg}$ ) tem sido associada com mortalidade perioperatória de $50-80 \%{ }^{10}$ e, naqueles com PMAP $\geq 50 \mathrm{mmHg}$, é de $100 \%$ e são considerados contraindicados. ${ }^{9}$

Candidatos a transplante de fígado são rotineiramente diagnosticados na fila de espera para hipertensão pulmonar moderada a grave em muitos centros transplantadores. O prognóstico da hipertensão portopulmonar não tratada é geralmente pobre e a taxa de sobrevida, em cinco anos, sem qualquer terapia é de $14 \%$. Já os pacientes que recebem terapia vasodilatadora têm tempo de sobrevida médio de 46 meses e taxa de sobrevida, em cinco anos, de $45 \% .^{11}$

O presente estudo objetivou relatar o caso de uma paciente portadora de HPP, que apresentou aumento súbito da PMAP durante a fase de reperfusão do transplante hepático.

\section{RELATO DE CASO}

Paciente do sexo feminino, 40 anos, $68 \mathrm{~kg}$, ASA IV, MELD 21 , capacidade funcional < $4 \mathrm{METs}$. Nos últimos três anos, passou a apresentar ascite, hepatoesplenomegalia, dispneia e prurido difuso, constatando-se hepatopatia alcóolica, hipertensão pulmonar, insuficiência renal crônica em hemodiálise, insuficiência cardíaca, sendo listada na fila de transplante de fígado e rim.
$\mathrm{Na}$ avaliação pré-anestésica, apresentou estado geral regular, orientada, cooperativa, eupnéica, acianótica, afebril, anictérica, normocorada, pressão arterial: $130 \times 70 \mathrm{mmHg}$, frequência cardíaca: $90 \mathrm{bpm}$, frequência respiratória: 15 irpm, Mallampati II, sem sinais de dificuldade para intubação.

Exames laboratoriais: hemoglobina $(\mathrm{Hb}): 15,7 \mathrm{~g} / \mathrm{dL}$; hematócrito (Ht): 47,2\%; plaquetas: 105000 /mm3; albumina: $4,5 \mathrm{mg} / \mathrm{dL}$; razão normalizada internacional (INR): 1,14; tempo de tromboplastina parcial ativada (TTPa): 1,24; creatinina (Cr): $8,4 \mathrm{mg} / \mathrm{dL}$; ureia (Ur): $108 \mathrm{mg} / \mathrm{dL} ; \mathrm{Na}+: 141 \mathrm{mg} / \mathrm{dL} ; \mathrm{K}+: 4,1 \mathrm{mg} / \mathrm{dL}$; bilirrubina indireta (BI): $0,45 \mathrm{mg} / \mathrm{dL}$; bilirrubina direta (BD): 0,20 $\mathrm{mg} / \mathrm{dL}$; bilirrubina total(BT): 0,65 mg/dL.

Exames auxiliares: Ecodopplercardiograma (28/11/2012): Pressão sistólica da artéria pulmonar estimado em 30 $\mathrm{mmHg}$, sendo indicado cateterismo cardíaco direito, que mostrou pressão da artéria pulmonar de $72 / 35 \mathrm{mmHg}$ com PMAP de $48 \mathrm{mmHg}$. Ecodopplercardiograma (03/04/2013): Dilatação moderada biatrial, hipertrofia concêntrica moderada de ventrículo esquerdo (VE), fração de ejeção de $64 \%$, disfunção diastólica do VE tipo pseudonormal, refluxo tricúspide mínimo, calcificação do anel mitral e regurgitação leve.

Paciente foi admitida na sala de operação para transplante hepático pela técnica de piggyback, após monitorização e indução de anestesia geral. O cateter de Swan-Ganz diagnosticou PMAP de $28 \mathrm{mmHg}$ e pressão venosa central (PVC) de $24 \mathrm{mmHg}$. Gasometria arterial do inicio do procedimento: $\mathrm{pH}:$ 7,41; PaO2: 200 mmHg; PaCO2: 33 mmHg; HCO3-: 22,1 mEq/L; BE: -3,4 $\mathrm{mEq} / \mathrm{L}$; SaO2: 99,7\%; Na+: $141 \mathrm{mEq} / \mathrm{L} ; \mathrm{K}+: 4,1 \mathrm{mEq} / \mathrm{L}$; Ca2+: 1,0 mEq/L; Hb: 14,4 g/dL; Ht: 44\%; Glicemia: 110 $\mathrm{mg} / \mathrm{dL}$.

A hidratação foi guiada pela variação de volume sistólico verificado no sistema EV1000 ${ }^{\mathrm{TM}}$ (Edwards Lifesciences, Irvine CA, USA) e pela variação de pressão pulmonar analisada com o cateter de Swan Ganz. Foi ministrado um total de $1650 \mathrm{ml}$ de solução albuminada (plasma lyte $1500 \mathrm{ml}+$ albumina $20 \% 150 \mathrm{ml}$ ).

Na fase anepática, a PMAP permaneceu sem alteração. Já na reperfusão hepática, foi registrada variação da PMAP de 40 a $50 \mathrm{mmHg}$ retornando ao valor basal após 45 minutos, como verificado na figura 1. Até esse momento, foram ministrados $900 \mathrm{ml}$ de solução albuminada a $2 \%$.

Gasometria após reperfusão: pH: 7,28; $\mathrm{PaO} 2: 191$ mmHg; PaCO2: 40 mmHg; HCO3-: 18,3 mEq/L; BE: -7,3 $\mathrm{mEq} / \mathrm{L}$; SaO2: 99,5\%; Na+: $142 \mathrm{mEq} / \mathrm{L} ; \mathrm{K}+: 3,8 \mathrm{mEq} / \mathrm{L}$; $\mathrm{Ca} 2+: 1,22 \mathrm{mEq} / \mathrm{L} ; \mathrm{Hb}: 14,7 \mathrm{~g} / \mathrm{dL}$; Ht: 45\%; Glicemia: $110 \mathrm{mg} / \mathrm{dL}$. Foram ministrados 60 meq de bicarbonato de sódio $8,4 \%$ em infusão contínua. 
José Carlos Rodrigues Nascimento, Aline Menezes Sampaio, Tayná de Lima Freire, Anderson Emanuel Caliope Castelo Branco Mourão, Antonio Tiago Mota Pinheiro, Felipe Barbosa Lima, David Silveira Marinho, Anderson da Silva Costa.

Gasometria arterial do final da cirurgia: $\mathrm{pH}: 7,30$; $\mathrm{PaO} 2$ : $172 \mathrm{mmHg}$; PaCO2: $46 \mathrm{mmHg}$; HCO3-: 21,1 mEq/L; BE: -3,5 mEq/L; SaO2: 99,4\%; Na: $141 \mathrm{mEq} / \mathrm{L} ; \mathrm{K}: 4,1 \mathrm{mEq} / \mathrm{L}$; Ca: $1,29 \mathrm{mEq} / \mathrm{L} ; \mathrm{Hb}: 14,3 \mathrm{~g} / \mathrm{dL} ; \mathrm{Ht}: 44 \%$; Glicemia: 196 $\mathrm{mg} / \mathrm{dL}$.

Nofinal da cirurgia, a paciente estavahemodinamicamente estável, com PMAP de $31 \mathrm{mmHg}$ e foi encaminhada à UTI. Foi submetida a transplante renal 24 horas após o transplante hepático e recebeu alta hospitalar sem complicações.

Figura 1: Pressões pulmonares no período transoperatório

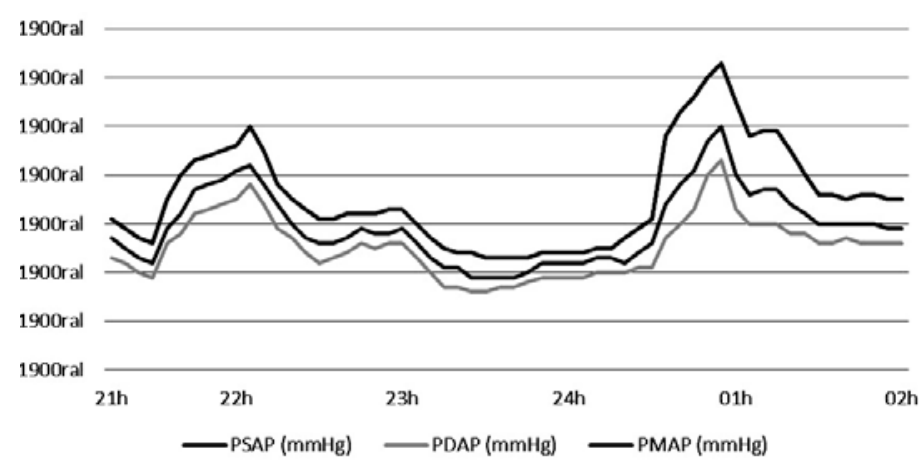

PSAP: Pressão sistólica arterial pulmonar;

PADP: Pressão diastólica arterial pulmonar;

PMAP: Pressão média na artéria pulmonar;

h: Horas

\section{DISCUSSÃO}

Há grande variação de prevalência de HPP dependendo da evidência, porém Starkel et al. verificaram sua presença em média de $25 \%$ dos pacientes com doença hepática avançada. ${ }^{10} \mathrm{~A}$ patogênese exata desse tipo de hipertensão pulmonar não está bem definida. A circulação hiperdinâmica arterial, o aumento do volume de sangue venoso e da pressão, a vasoconstricção da artéria pulmonar e a ativação neuro-humoral são responsáveis pelo desenvolvimento de HPP em pacientes cirróticos. ${ }^{11}$

Apesar de ter sido detectada uma PMAP de $48 \mathrm{mmHg}$ em exame de cateterismo cardíaco, como descrito anteriormente, não houve tratamento prévio ao procedimento cirúrgico. Como foi verificada PMAP de $28 \mathrm{mmHg}$ após a monitorização com o cateter de SwanGanz, sendo classificada de grau leve e com pequeno risco perioperatório, ${ }^{9,10}$ optou-se pela realização do transplante hepático.

$\mathrm{Na}$ fase anepática, o clampeamento da porta e da veia cava inferior contribuiu para a redução da précarga juntamente com as drogas anestésicas, havendo redução da PMAP, que variou de 21 a $23 \mathrm{mmHg}$. Porém, na reperfusão, ocorreu aumento súbito da PMAP, variando de 26 até $50 \mathrm{mmHg}$, retornando a 30 $\mathrm{mmHg}$, após 40 minutos pós-reperfusão e, como a HPP sobrecarrega o ventrículo direito e induz mecanismos hemodinâmicos de adaptação, foram implementadas medidas de restrinção volêmica, sem o uso de droga vasodilatadora pulmonar, para evitar a sobrecarga cardíaca durante a reperfusão, diminuindo ou abolindo as consequências potencialmente fatais, decorrentes do aumento da pressão arterial pulmonar. ${ }^{12}$

Neste caso, o aumento súbito da PMAP na reperfusão assemelhou-se ao estudo de Taura et al., onde foi observado aumento súbito da pressão arterial pulmonar e pressão venosa central após a reperfusão do fígado, devido ao aumento da pré-carga, apesar de terem usado droga vasodilatadora pulmonar no intraoperatório, mas sem aumento da morbidade e mortalidade durante 0 período perioperatório. ${ }^{13}$

No estudo de De Wolf et al. foi relatado que, durante a fase anepática, há ligeira diminuição da fração de ejeção do ventrículo direito e também do índice de volume diastólico e sistólico final. ${ }^{14}$

Yassen et al. observaram que a fração de ejeção do ventrículo direito foi menor em pacientes com HPP leve a moderada em relação ao controle e que a hemodinâmica do ventrículo direito deteriorou-se após a reperfusão no grupo HPP, mas não houve morbidade ou mortalidade significativa após o transplante hepático. ${ }^{15}$

Estratégia de infusão de líquidos continua a ser questão de debate. A abordagem padrão para o paciente com hipotensão presumida devido à hemorragia, foi infundir grandes volumes de fluido tão cedo e tão rapidamente quanto possível, a fim de restaurar o volume intravascular e estabilidade hemodinâmica para manter a perfusão dos órgãos vitais. No entanto, alguns estudos relataram que a infusão agressiva de fluido pode ser prejudicial, resultando em aumento da perda de sangue $e$, consequentemente, maior mortalidade, e que a infusão de volume restrito pode ser preferível. ${ }^{16}$

Neste relato de caso, a restrição volêmica seguida pela variação de volume sistólico e PMAP associada à não necessidade de transfusão sanguínea, permitiram dispensar técnicas especiais para evitar transfusões sanguíneas, mesmo com alto potencial de sangramento do procedimento cirúrgico, evitando assim, sobrecarga cardíaca, congestão do enxerto e complicações pulmonares em uma paciente com hipertensão pulmonar.

Fujita et al. mostraram redução da perda de sangue com estratégia de restrição de volume de fluido em hepatectomia de doador vivo para transplante hepático. ${ }^{17}$ 
O estudo de Snowden et al. demonstrou que, em algumas circunstâncias, o aumento da necessidade de transfusão pode ter um papel no desenvolvimento de edema pulmonar pós-operatório imediato, aumentando a pressão pulmonar e promovendo a lesão pulmonar aguda, levando à alta incidência de edema pulmonar, associada à troca prejudicada de gases, aumento na necessidade de ventilação e permanência prolongada em unidade de terapia intensiva. ${ }^{18}$

Perilli et al. evidenciaram que a normovolemia deve ser o ponto final de uma estratégia de substituição de fluidos em ambiente de transplante de fígado, evitando baixos índices de pré-carga em receptores de fígado, pelo menos, após a reperfusão do enxerto para evitar disfunção do enxerto no pós-operatório imediato. ${ }^{19}$
Cywinski et al. concluíram que a manutenção da pressão venosa central mais baixa durante a fase pós-anepática, não está associada a qualquer benefício em termos de função do enxerto no pós-operatório imediato, na sobrevida do enxerto ou na sobrevida do paciente. ${ }^{20}$

Este relato de caso mostrou o aumento súbito da PMAP durante a reperfusão, apesar de terem sido adotadas medidas de restrição da infusão de fluidos no intraoperatório, para evitar o aumento da pressão arterial pulmonar, decorrente da sobrecarga volêmica.

No entanto, mesmo neste caso, não havendo aumento da morbimortalidade no perioperatório, essa medida de restrição volêmica adotada sem o uso de droga vasodilatadora pulmonar, não foi capaz de prevenir o aumento súbito da PMAP durante a fase de reperfusão do transplante hepático.

\section{ABSTRACT}

Introduction: The existence of pulmonary hypertension associated to portal hypertension is a relevant condition in cirrhotic patients. In the severe form, it is an absolute contraindication for liver transplantation. We report a case of portopulmonary hypertension in liver transplantation with severe hypertensive peak during reperfusion of liver graft. Case report: Female patient, 40-year-old, functional capacity <4 METs, ASA IV, bearer of alcoholic liver disease with portopulmonary hypertension, dialysis kidney failure and heart failure. Doppler echocardiography showed systolic pulmonary artery pressure estimated at $30 \mathrm{mmHg}$, and indicated right heart catheterization. This showed $48 \mathrm{mmHg}$ mean pulmonary arterial pressure (MPAP). Patient underwent liver transplantation under general anesthesia and monitored with a Swan-Ganz ${ }^{\circledR}$ catheter, showing MPAP $28 \mathrm{mmHg}$ and $24 \mathrm{mmHg}$ central venous pressure. The gasometry at hepatectomy was normal. The anhepatic phase had not any considerable bleeding, and slight MPAP oscillation. In the reperfusion, it was recorded a greatest MPAP variation $(26-50 \mathrm{mmHg})$, which lasted 40 minutes until returning to the baseline with expectant management. Until then, they were given $900 \mathrm{ml}$ of plasma-lyte albuminated $2 \%$ solution. The arterial blood gas after reperfusion presented metabolic and respiratory acidosis with $7.28 \mathrm{pH}$. At the end of the surgery, patient was hemodynamically stable with MPAP $30 \mathrm{mmHg}$. She was referred to the ICU intubated. The total volume infused was $1650 \mathrm{ml}$. The patient underwent a kidney transplant 24 hours after liver transplantation, and she was discharged without complications. Discussion: The intraoperative anesthetic management requires knowledge of the variations of pulmonary artery pressure in each stage of liver transplantation. Proper monitoring of cardiac output, stroke volume variation and pulmonary artery pressures allowed the optimization of using vasoactive drugs fluid replacement. Although water restriction is described as an adequate way to control the volume overload in the hepatic reperfusion phase, which is a factor of cardiovascular instability in those patients, in this case, the measurement of the volume restriction adopted, without using pulmonary vasodilator drug was unable to prevent the sudden increase of MPAP in the reperfusion of the graft.

Keywords: Pulmonary Hypertension; Portal Hypertension; Liver Transplantation. 
José Carlos Rodrigues Nascimento, Aline Menezes Sampaio, Tayná de Lima Freire, Anderson Emanuel Caliope Castelo Branco Mourão, Antonio Tiago Mota Pinheiro, Felipe Barbosa Lima, David Silveira Marinho, Anderson da Silva Costa.

\section{REFERÊNCIAS}

1. Simonneau G, Gatzoulis MA, Adatia I, Celermajer D, Denton C, Ghofrani A et al. Updated clinical classification of pulmonar hypertension. J Am Coll Cardiol. 2013;62 Suppl 25:S34-41.

2. Ramsay MAE, Schmidt A, Hein HA, Nguyen AT, Lynch $\mathrm{K}$, East CA et al. Nitric oxide does not reverse pulmonary hypertension associated with end-stage liver disease: a preliminary report. Hepatology. 1997;25:524-7.

3. Menna Barreto SS, Gazzana MB. Hipertensão Pulmonar: relato de seis casos e atualização do tema. Jornal de Pneumologia. 2000;26:321-36.

4. Almeida DR, Diniz RV, Areosa C, Ota JS, Viegas RF, Silva AC et al. Hipertensão Pulmonar Primária. Estado de São Paulo. Rev Soc Cardiol. 2000(set/out);10:576-89.

5. Badesch DB, Raskob GE, Elliott CG, Krichman AM, Farber HW, Frost AE et al. Pulmonary arterial hypertension: baseline characteristics from the REVEAL Registry. Chest. 2010;137:376-87.

6. Wells JT, Runo JR, Lucey MR. Portopulmonary hypertension. Hepatology. 2008;48:13-5.

7. Krowka MJ, Miller DP, Barst RJ, Taichman D, Dweik RA, Badesch DB et al. Portopulmonary hypertension: a report from the US-based REVEAL registry. Chest. 2012;141:906-15.

8. Sitbon $O$, Yaïci A, Cottin $V$ et al. The changing picture of patients with pulmonary arterial hypertension in France. Eur Heart J. 2011; 32 Suppl 1:S675-6.

9. Ayoub T. Pulmonary hypertension in liver transplant. Current Opinion in Organ Transplantation. 2011;16:331-337.

10.Starkel P, Vera A, Gunson B, Mutimer D. Outcome of liver transplantation for patients with pulmonary hypertension. Liver Transpl. 2002;8:382-8.

11.Swanson KL, Wiesner RH, Nyberg SL, Rosen CB, Krowka MJ. Survival in portopulmonary hypertension: Mayo Clinic experience categorized by treatment subgroups. Am J Transplant. 2008;8:2445-53.

12.Bozbas SS, Bozbas H. Portopulmonary hypertension prior to liver transplantation. World J Gastroenterol. 2016;22(6):2024-9.
13.Taura P, Garcia-Valdecasas JC, Beltran J, Izquierdo E, Navasa M, Sala-Blanch J et al. Moderate Primary Pulmonary Hypertension in Patients Undergoing liver Transplantation. Anesth Analg. 1996;83:675-8.

14.De Wolf AM, Begliomini B, Gasior TA, Kang Y, Pinsky MR. Right ventricular function during orthotopic liver transplantation. Anesth Analg. 1993;76:562-8.

15.Yassen AM, Elsarraf WR, Elsadany M, Elshobari MM, Salah T, Sultan AM. The impact of portopulmonary hypertension on intraoperative right ventricular function of living donor liver transplant recipients. Anesth Analg. 2012;115: 689-93.

16.Bickell WH, Bruttig SP, Millnamow GA, O’Benar J, Wade CE. Use of hypertonic saline/dextran versus lactated Ringer's solution as a resuscitation fluid after uncontrolled aortic hemorrhage in anesthetized swine. Ann Emerg Med. 1992;21:1077-85.

17.Fujita Y, Takeuchi A, Sugiura T, Hattori T, Sasano N, Mizuochi $Y$ et al. Before-after study of a restricted fluid infusion strategy for management of donor hepatectomy for living-donor liver transplantation. J Anesth. 2009;23(1):67-74.

18.Snowden CP, Hughes T, Rose J, Roberts DR. Pulmonary edema in patients after liver transplantation. Liver Transplantation. 2000;4:466-70.

19.Perilli V, Aceto P, Modesti C, Ciocchetti P, Sacco T, Vitale $F$ et al. Low values of left ventricular ejection time in the post-anhepatic phase may be associated with occurrence of primary graft dysfunction after orthotopic liver transplantation: results of a single centre case control study. European Review for Medical and Pharmacological Sciences. 2012;16:1433-40.

20.Cywinski JB, Mascha E, You J, Argalious M, Kapural $\mathrm{L}$, Christiansen $\mathrm{E}$ et al. Central venous pressure during the post-anhepatic phase is not associated with early postoperative outcomes following orthotopic liver transplantation. Minerva Anestesiol. 2010;76:795804. 